\title{
Resonant field enhancement in lossy periodic structures supporting complex bound states in the continuum
}

\author{
Ling Tan and Lijun Yuar* \\ College of Mathematics and Statistics, Chongqing Technology and Business University, Chongqing, China \\ Chongqing Key Laboratory of Social Economic and Applied Statistics, \\ Chongqing Technology and Business University, Chongqing, China \\ Ya Yan $\mathrm{Lu}$ \\ Department of Mathematics, City University of Hong Kong, Kowloon, Hong Kong, China
}

(Dated: August 13, 2021)

\begin{abstract}
Resonant modes in a lossy periodic structure sandwiched between two lossless homogeneous media form bands that depend on the Bloch wavevector continuously and have a complex frequency due to radiation and absorption losses. A complex bound state in the continuum (cBIC) is a special state with a zero radiation loss in such a band. Plane waves incident upon the periodic structure induce local fields that are resonantly enhanced. In this paper, we derive a rigorous formula for field enhancement, and analyze its dependence on the frequency, wavevector and amplitude of the incident wave. For resonances with multiple radiation channels, we determine the incident wave that maximizes the field enhancement, and find conditions under which the field enhancement can be related to the radiation and dissipation quality factors. We also show that with respect to the Bloch wavevector, the largest field enhancement is obtained approximately when the radiation and dissipation quality factors are equal. Our study clarifies the various factors related to field enhancement, and provides a useful guideline for applications where a strong local field is important.
\end{abstract}

\section{INTRODUCTION}

A strong local field is important to many applications in photonics, such as sensing [1 [4] and imaging [5, 6], and is essential to the enhnancement of emissive processes and nonlinear optical effects [7-13]. For structures supporting a high quality factor ( $Q$ factor) resonance, if the frequency of the incident wave is close to the resonant frequency, the amplitude of the local field can be much larger than that of the incident wave [1419]. The strength of resonant field enhancement, i.e. the ratio of the amplitudes of the local field and the incident wave, depends on the resonant mode and its coupling with the incident wave 7, 14, 17]. For unbounded structures that are periodic or invariant in at least one spatial direction, resonant modes form bands that vary with the wavevector continuously. In recent years, dielectric periodic structures supporting bound states in the continuum (BICs) have been intensively studied [20 28]. In a lossless periodic structure, a BIC is a special bound state in a band of resonant modes. As the wavevector of the resonant modes tends to that of the BIC, the $Q$ factor diverges [20], and in principle, the field enhancement can be arbitrarily large [17].

However, electromagnetic waves in practical materials always suffer from absorption loss (dissipation). For a lossy periodic structure surrounded by homogeneous and lossless dielectric media, any eigenmode with a real wavevector must have a complex frequency. A bound state is an eigenmode that decays exponentially in the

\footnotetext{
*ljyuan@ctbu.edu.cn
}

surrounding homogeneous media. If the real part of its complex frequency lies in the radiation continuum, the bound state will be referred to as a complex BIC (cBIC) in this paper. For periodic structures with an in-plane inversion symmetry, symmetry-protected cBICs are easy to find 29]. A resonant mode is an eigenmode that radiates out power in the surrounding media. In a lossy structure, the total $Q$ factor $\left(Q_{\mathrm{tot}}\right)$ of a resonant mode satisfies $1 / Q_{\text {tot }}=1 / Q_{\text {rad }}+1 / Q_{\text {dis }}$, where $Q_{\text {rad }}$ and $Q_{\text {dis }}$ are the $Q$ factors associated with radiation and absorption losses, respectively 30]. In a lossy periodic structure, if there is a cBIC in a band of resonant modes, then $Q_{\text {rad }} \rightarrow \infty$ and $Q_{\text {dis }}$ remains bounded, as the wavevector tends to that of the cBIC. It has been shown that field enhancement is related to $Q_{\mathrm{rad}}$ and $Q_{\mathrm{dis}}$, and the maximum field enhancement is obtained at the wavevector satisfying $Q_{\mathrm{rad}}=Q_{\mathrm{dis}}$ (the critical coupling condition) [14, 31] .

The existing results on resonant field enhancement are derived from the temporal coupled mode theory (CMT) [7, 14. The CMT is a powerful modeling technique that allows one to reveal the most important physical phenomena. It relies on the assumption that the field inside the "cavity" is exactly a resonant mode and the field outside the "cavity" is exactly the sum of an incoming wave and an outgoing wave. The actual field satisfying the Maxwell's equations is of course far more complicated. In this paper, we use a perturbation method to derive an accurate formula for field enhancement. It reveals that field enhancement depends on the resonant mode, its coupling with the incident wave, and the frequency of the incident wave. We pay special attention to the case where the resonant mode has multiple radiation channels, determine the incident wave that maximize the field enhancement, and show that field enhancement 
is proportional to $\sqrt{Q_{\mathrm{rad}}} Q_{\mathrm{dis}} /\left(Q_{\mathrm{rad}}+Q_{\mathrm{dis}}\right)$ only for special (usually non-optimal) incident waves. We also study the dependence on wavevector for structures supporting a cBIC, and confirm that the largest field enhancement is obtained at a wavevector satisfying the the critical coupling condition approximately.

The rest of this paper is organized as follows. In Sec.II. we give some background material on resonant modes and cBICs in lossy periodic structures, and present some integral formulas for $Q_{\mathrm{rad}}$ and $Q_{\mathrm{dis}}$. In Sec. III we drive a rigorous formula for field enhancement by a perturbation method, find the conditions such that the field enhancement is proportional to $\sqrt{Q_{\mathrm{rad}}} Q_{\mathrm{dis}} /\left(Q_{\mathrm{rad}}+Q_{\mathrm{dis}}\right)$, and determine incident waves that maximize the field enhancement. Numerical examples are presented in Sec.IV] to illustrate how field enhancement depends on the incident wave and the Bloch wavenumber. The paper is concluded with a brief discussion in Sec. V

\section{RESONANT AND BOUND STATES}

We consider a lossy two-dimensional (2D) structure that is invariant in $z$, symmetric and periodic (with period $L$ ) in $y$, and sandwiched between two identical lossless homogeneous media given for $x>d$ and $x<-d$, respectively. The dielectric function satisfies

$$
\varepsilon(\mathbf{r})=\varepsilon(x, y+L)=\varepsilon(x,-y)
$$

and $\operatorname{Im}(\varepsilon) \geq 0$ for all $\mathbf{r}=(x, y), \max [\operatorname{Im}(\varepsilon)]>0$, and $\varepsilon(\mathbf{r})=\varepsilon_{0} \geq 1$ for $|x|>d$. For any $E$-polarized timeharmonic wave with the time dependence $e^{-i \omega t}(\omega$ is the angular frequency), the $z$-component of the electric field, denoted as $u(\mathbf{r})$, satisfies the following 2D Helmholtz equation

$$
\partial_{x}^{2} u+\partial_{y}^{2} u+k^{2} \varepsilon(\mathbf{r}) u=0,
$$

where $k=\omega / c$ is the freespace wave number and $c$ is the speed of light in vacuum.

Due to the periodicity, any eigenmode of the structure is a Bloch mode given as

$$
u(\mathbf{r})=\phi(\mathbf{r}) e^{i \beta y},
$$

where $\phi$ is periodic in $y$ and $\beta$ is the Bloch wavenumber. We assume $\beta$ is real and $\omega$ (or $k$ ) is the eigenvalue. A resonant mode (or resonant state) is an eigenmode satisfying outgoing radiation conditions. It radiates out power and diverges as $x \rightarrow \pm \infty$. In contrast, a bound state decays to zero exponentially as $x \rightarrow \pm \infty$. Since the structure is lossy, $\operatorname{Im}(\omega)$ is negative for both resonant and bound states. In the surrounding homogeneous media, the Bloch mode can be expanded as

$$
u(\mathbf{r})=\sum_{m=-\infty}^{\infty} c_{m}^{ \pm} e^{i\left[\beta_{m} y \pm \alpha_{m}(x \mp d)\right]}, \quad \pm x>d,
$$

where $c_{m}^{ \pm}$, for all integers $m$, are expansion coefficients, $\beta_{0}=\beta$, and

$$
\beta_{m}=\beta+2 m \pi / L, \quad \alpha_{m}=\sqrt{k^{2} \varepsilon_{0}-\beta_{m}^{2}} .
$$

In order for Eq. (4) to describe both resonant and bound states correctly, we need to choose the complex square root in Eq. (5) to maintain continuity as $\operatorname{Im}(\omega) \rightarrow 0$. More precisely, if $\operatorname{Re}\left(k^{2} \varepsilon_{0}-\beta_{m}^{2}\right)$ is positive, then $\alpha_{m}$ is in the fourth quadrant of the complex plane with $\operatorname{Re}\left(\alpha_{m}\right)>$ 0 and $\operatorname{Im}\left(\alpha_{m}\right)<0$, so that $e^{i \alpha_{m} x}$ is an outgoing plane wave (with increasing amplitude) as $x \rightarrow+\infty$; and if $\operatorname{Re}\left(k^{2} \varepsilon_{0}-\beta_{m}^{2}\right)$ is negative, then $\alpha_{m}$ is chosen to be in the second quadrant with $\operatorname{Re}\left(\alpha_{m}\right)<0$ and $\operatorname{Im}\left(\alpha_{m}\right)>0$, so that $e^{i \alpha_{m} x}$ decays exponentially as $x \rightarrow+\infty$. We denote the set of integers $m$ such that $\operatorname{Re}\left(k^{2} \varepsilon_{0}-\beta_{m}^{2}\right)>$ 0 by $\mathbb{Z}_{0}$. If $\mathbb{Z}_{0}$ is not empty and there is at least one nonzero coefficient $c_{m}^{+}$or $c_{m}^{-}$for an $m \in \mathbb{Z}_{0}$, then the eigenmode is a resonant mode that radiates out power. If $\mathbb{Z}_{0}$ is not empty, but $c_{m}^{ \pm}=0$ for all $m \in \mathbb{Z}_{0}$, then the eigenmode is a cBIC. We assume $\beta$ has been shifted to satisfy $|\beta| \leq \pi / L$, then a non-empty $\mathbb{Z}_{0}$ implies that $0 \in \mathbb{Z}_{0}$, i.e., $\operatorname{Re}\left(k^{2} \varepsilon_{0}-\beta^{2}\right)>0$. If $|\operatorname{Im}(\omega) / \omega|$ is small, this condition can be approximated by $\operatorname{Re}(k) \sqrt{\varepsilon_{0}}>|\beta|$.

For a resonant mode with a complex frequency $\omega$, the total $Q$ factor may be defined by $Q_{\text {tot }}=$ $-0.5 \operatorname{Re}(\omega) / \operatorname{Im}(\omega)$. Meanwhile, according to Eq. (4), we can decompose the field into radiating and evanescent components as

$$
u(\mathbf{r})=u_{r}(\mathbf{r})+u_{e}(\mathbf{r}), \quad|x|>d,
$$

where

$$
u_{e}(\mathbf{r})=\sum_{m \notin \mathbb{Z}_{0}} c_{m}^{ \pm} e^{i\left[\beta_{m} y \pm \alpha_{m}(x \mp d)\right]}, \quad|x|>d,
$$

and $u_{r}$ is given similarly for $m \in \mathbb{Z}_{0}$. In Appendix A, we prove that

$$
\frac{1}{Q_{\mathrm{tot}}}=\frac{1}{Q_{\mathrm{dis}}}+\frac{1}{Q_{\mathrm{rad}}}
$$

where

$$
\begin{aligned}
& \frac{1}{Q_{\mathrm{dis}}}=\frac{\operatorname{Re}\left(k^{2}\right) \int_{\Omega_{d}} \varepsilon_{i}(\mathbf{r})|u(\mathbf{r})|^{2} d \mathbf{r}}{[\operatorname{Re}(k)]^{2} F}, \\
& \frac{1}{Q_{\mathrm{rad}}}=\frac{L \sum_{m \in \mathbb{Z}_{0}}\left(\left|c_{m}^{-}\right|^{2}+\left|c_{m}^{+}\right|^{2}\right) \operatorname{Re}\left(\alpha_{m}\right)}{[\operatorname{Re}(k)]^{2} F}, \\
& F=\int_{\Omega_{d}} \varepsilon_{r}(\mathbf{r})|u(\mathbf{r})|^{2} d \mathbf{r}+\varepsilon_{0} \int_{\Omega_{e}}\left|u_{e}(\mathbf{r})\right|^{2} d \mathbf{r} .
\end{aligned}
$$

In the above, $\varepsilon_{r}(\mathbf{r})$ and $\varepsilon_{i}(\mathbf{r})$ are the real and imaginary parts of the dielectric function $\varepsilon(\mathbf{r}), \Omega_{d}$ is the rectangular domain given by $|x|<d$ and $|y|<L / 2, \Omega_{e}$ is the union of two semi-infinite strips given by $|x|>d$ and $|y|<$ $L / 2$. The first and second integrals in the expression $F$ are proportional to the energy stored in domain $\Omega_{d}$ (i.e. the cavity) and the energy of the evanescent field 
$u_{e}$ outside domain $\Omega_{d}$, respectively. The numerator in Eq. (8) is proportional to the power absorbed by material dissipation. The numerator in Eq. (9) is proportional to the power radiated out as $x \rightarrow \pm \infty$. The above equations give the well-known interpretation that $1 / Q$ is the ratio of power lost per cycle and total stored energy, $Q_{\text {dis }}$ and $Q_{\text {rad }}$ are quality factors associated with absorption and radiation losses, respectively [30]. The imaginary part of $\omega$ can also be decomposed as

$$
\operatorname{Im}(\omega)=\omega_{\mathrm{dis}}^{\prime \prime}+\omega_{\mathrm{rad}}^{\prime \prime}
$$

where $\omega_{\text {dis }}^{\prime \prime}$ and $\omega_{\text {rad }}^{\prime \prime}$ are associated with dissipation and radiation losses, respectively, and satisfy

$$
Q_{\mathrm{dis}}=-\frac{\operatorname{Re}(\omega)}{2 \omega_{\mathrm{dis}}^{\prime \prime}} \quad \text { and } \quad Q_{\mathrm{rad}}=-\frac{\operatorname{Re}(\omega)}{2 \omega_{\mathrm{rad}}^{\prime \prime}}
$$

If the resonant mode is scaled such that

$$
\max _{\mathbf{r} \in \Omega_{d}}|u(\mathbf{r})|=1
$$

then $c_{m}^{ \pm}$in Eq. (41) are dimensionless constants, $F$ is proportional to $L^{2}, k$ and $\alpha_{m}$ are proportional to $1 / L$. Therefore, Eq. (9) leads to

$$
\frac{1}{Q_{\mathrm{rad}}} \sim \frac{1}{\operatorname{Re}(k)} \sum_{m \in \mathbb{Z}_{0}}\left(\left|c_{m}^{-}\right|^{2}+\left|c_{m}^{+}\right|^{2}\right) \operatorname{Re}\left(\alpha_{m}\right)
$$

A cBIC is a special point in a branch of resonant modes. It can be regarded as a special resonant mode with $Q_{\text {rad }}=\infty$ (thus $\left.Q_{\text {tot }}=Q_{\text {dis }}\right)$. Let $\beta_{\diamond}$ be the real Bloch wavenumber of the $\mathrm{cBIC}$, then $Q_{\mathrm{rad}}$ of the resonant mode with Bloch wavenumber $\beta$ tends to infinity as $\beta \rightarrow \beta_{\diamond}$. In the same limit, $Q_{\text {dis }}$ of the resonant mode tends to a finite constant, i.e., the $Q_{\text {dis }}$ of the cBIC.

\section{FIELD ENHANCEMENT}

In this section, we analyze field enhancement for incident waves impinging upon a lossy periodic structure. Using a perturbation method, we derive an explicit formula for field enhancement, study how it depends on the incident waves and the $Q$ factors. In the case of multiple open diffraction channels, we specify a condition on the incident wave for maximum field enhancement. It is also shown that when the periodic structure supports a cBIC, the largest field enhancement occurs approximately at the wavenumber satisfying the critical coupling condition, i.e., $Q_{\mathrm{rad}}=Q_{\mathrm{dis}}$.

We consider a $2 \mathrm{D}$ lossy symmetric periodic structure as described in Sec. II assume that there is a resonant mode $u_{*}$ with a complex frequency $\omega_{*}$ depending on the real Bloch wavenumber $\beta$, and expand $u_{*}$ as in Eq. (44) with $\alpha_{m}$ replaced by $\alpha_{m}^{*}=\sqrt{k_{*}^{2} \varepsilon_{0}-\beta_{m}^{2}}$ for $k_{*}=\omega_{*} / c$. For the given $\beta$ and a real frequency $\omega$ close or equal to
$\operatorname{Re}\left(\omega_{*}\right)$, we study the diffraction problem with an incident wave given by

$$
u^{(i n)}(\mathbf{r})=\sum_{m \in \mathbb{Z}_{0}} a_{m}^{ \pm} e^{i\left[\beta_{m} y \mp \alpha_{m}(x \mp d)\right]}, \quad \pm x>d,
$$

where $a_{m}^{ \pm}$(for $m \in \mathbb{Z}_{0}$ ) are the coefficients of incident plane waves, $\beta_{m}$ and $\alpha_{m}$ are given in Eq. (5), $\mathbb{Z}_{0}$ is the set of all propagating diffraction orders (or open diffraction channels) and is defined in the previous section. Notice that $u^{(i n)}$ consists of incoming plane waves given in the left $(x<-d)$ and right $(x>d)$ sides of the periodic structure. We further assume that the coefficients of the incident plane waves satisfy

$$
\max \left\{\sum_{m \in \mathbb{Z}_{0}}\left|a_{m}^{-}\right|, \sum_{m \in \mathbb{Z}_{0}}\left|a_{m}^{+}\right|\right\}=1
$$

This implies that the amplitude of $u^{(i n)}$ is bounded by one, i.e. $\left|u^{(i n)}(\mathbf{r})\right| \leq 1$ for all $\mathbf{r} \in \Omega_{e}$.

Let $u$ be the diffraction solution associated with the incident wave given above. If $\left|\omega-\omega_{*}\right| /\left|\omega_{*}\right|$ is small, we expand $u$ in a Laurent series of $\delta=k-k_{*}$ :

$$
u=\frac{C}{\delta} u_{*}+u_{0}+\delta u_{1}+\ldots,
$$

where $C$ is a constant. In Appendix B, we show that

$$
C=\frac{i L}{k_{*} R} \sum_{m \in \mathbb{Z}_{0}}\left(a_{m}^{-} c_{m}^{-}+a_{m}^{+} c_{m}^{+}\right) \alpha_{m}^{*}
$$

where

$$
R=\int_{\Omega_{d}} \varepsilon(\mathbf{r}) u_{*}(x,-y) u_{*}(\mathbf{r}) d \mathbf{r}+\frac{i L}{2} \sum_{m=-\infty}^{+\infty} \frac{\left(c_{m}^{-}\right)^{2}+\left(c_{m}^{+}\right)^{2}}{\alpha_{m}^{*}}
$$

In the above, $c_{m}^{ \pm}$are the expansion coefficients of $u_{*}$ as given in Eq. (4). The sum in the right hand side of Eq. (17) provides the coupling between the resonant mode and the incident wave. Since the structure has a reflection symmetry in $y$, the resonant mode reciprocal to $u_{*}$, with the Bloch factor $e^{-i \beta y}$, is simply $u_{*}(x,-y)$, and it appears in the expression $R$.

The field enhancement factor (or field enhancement), denoted by $\eta$, can be defined as the ratio of maximum amplitudes of total field in $\Omega_{d}$ and the incident wave in $\Omega_{e}$, i.e.

$$
\eta=\frac{\max _{\mathbf{r} \in \Omega_{d}}|u(\mathbf{r})|}{\max _{\mathbf{r} \in \Omega_{e}}\left|u^{(i n)}(\mathbf{r})\right|} .
$$

Since $u_{*}$ and $u^{(i n)}$ are required to satisfy Eqs. (12) and (15), respectively, we have

$$
\eta \approx \max _{\mathbf{r} \in \Omega_{d}}|u(\mathbf{r})| \approx \frac{|C|}{|\delta|} .
$$


From Eq. (17), it is clear that field enhancement depends strongly on the incident wave. If the coefficients are chosen such that $\left(a_{m}^{-}, a_{m}^{+}\right)$is proportional to $\left(-c_{m}^{+}, c_{m}^{-}\right)$for each $m \in \mathbb{Z}_{0}$, then $C=0$ and there is no enhancement. On the other hand, if

$$
a_{m}^{ \pm}=B \frac{\bar{\alpha}_{m}^{*} \operatorname{Re}\left(\alpha_{m}^{*}\right)}{\left|\alpha_{m}^{*}\right|^{2}} \bar{c}_{m}^{ \pm}
$$

where $B$ is a constant chosen to satisfy Eq. (15), $\bar{\alpha}_{m}^{*}$ and $\bar{c}_{m}^{ \pm}$are the complex conjugates of $\alpha_{m}^{*}$ and $c_{m}^{ \pm}$, respectively, then

$$
\sum_{m \in \mathbb{Z}_{0}}\left(a_{m}^{-} c_{m}^{-}+a_{m}^{+} c_{m}^{+}\right) \alpha_{m}^{*}=B \sum_{m \in \mathbb{Z}_{0}}\left(\left|c_{m}^{-}\right|^{2}+\left|c_{m}^{+}\right|^{2}\right) \operatorname{Re}\left(\alpha_{m}^{*}\right) .
$$

In that case, using Eq. (13) (with $k$ and $\alpha_{m}$ replaced by $k_{*}$ and $\alpha_{m}^{*}$, respectively), we obtain

$$
|C| \sim \frac{1}{L \sqrt{Q_{\mathrm{rad}}}} .
$$

In addition, if we let $\omega=\operatorname{Re}\left(\omega_{*}\right)$, then $\delta=-\operatorname{Im}\left(k_{*}\right) \sim$ $1 /\left(L Q_{\text {tot }}\right)$, and thus

$$
\eta \sim \frac{Q_{\mathrm{dis}} \sqrt{Q_{\mathrm{rad}}}}{Q_{\mathrm{rad}}+Q_{\mathrm{dis}}}
$$

Notice that Eq. (22) is useful when parameters are introduced so that $Q_{\mathrm{rad}}$ and/or $Q_{\text {dis }}$ vary rapidly with the parameters, but the resonant mode is nearly invariant. For example, Eq. (22) is valid when $\operatorname{Im}(\varepsilon)$ is close to zero. $\operatorname{As} \operatorname{Im}(\varepsilon) \rightarrow 0, Q_{\text {dis }} \rightarrow \infty$ and $\eta \sim \sqrt{Q_{\text {rad }}}$. It is also valid when there is a $\mathrm{cBIC}$ and $\beta$ is close to that of the cBIC.

If the zeroth diffraction order is the only propagating one, i.e., $\mathbb{Z}_{0}=\{0\}$, and if $\left|c_{0}^{-}\right|=\left|c_{0}^{+}\right|$, then formula (22) gives the maximum field enhancement for optimally chosen incident waves. If $\mathbb{Z}_{0} \neq\{0\}$, and if $\mathbb{Z}_{0}=\{0\}$ but $\left|c_{0}^{-}\right| \neq\left|c_{0}^{+}\right|$, then a larger field enhancement can be obtained by appropriately choosing the coefficients of the incident wave. Let $m_{1}$ and $m_{2}$ be integers in $\mathbb{Z}_{0}$ that attach the maxima of $\left|\alpha_{m}^{*} c_{m}^{-}\right|$and $\left|\alpha_{m}^{*} c_{m}^{+}\right|$for all $m \in \mathbb{Z}_{0}$, respectively, that is,

$$
\left|\alpha_{m_{1}}^{*} c_{m_{1}}^{-}\right|=\max _{m \in \mathbb{Z}_{0}}\left|\alpha_{m}^{*} c_{m}^{-}\right|, \quad\left|\alpha_{m_{2}}^{*} c_{m_{2}}^{+}\right|=\max _{m \in \mathbb{Z}_{0}}\left|\alpha_{m}^{*} c_{m}^{+}\right| .
$$

Under the normalization condition (15), the maximum of $|C|$ is obtained when the incident wave in the left (for $x<-d)$ is a single plane wave of diffraction order $m_{1}$ with unit magnitude $\left(\left|a_{m_{1}}^{-}\right|=1\right)$, the incident wave in the right (for $x<d$ ) is a single plane wave of diffraction order $m_{2}$ with unit magnitude $\left(\left|a_{m_{2}}^{+}\right|=1\right)$, and when the phases of $a_{m_{1}}^{-}$and $a_{m_{2}}^{+}$are chosen such that

$$
\left|a_{m_{1}}^{-} c_{m_{1}}^{-} \alpha_{m_{1}}^{*}+a_{m_{2}}^{+} c_{m_{2}}^{+} \alpha_{m_{2}}^{*}\right|=\left|c_{m_{1}}^{-} \alpha_{m_{1}}^{*}\right|+\left|c_{m_{2}}^{+} \alpha_{m_{2}}^{*}\right| .
$$

This implies that the maximum of $|C|$ is

$$
|C|_{\max }=\frac{L}{\left|k_{*} R\right|}\left(\left|c_{m_{1}}^{-} \alpha_{m_{1}}^{*}\right|+\left|c_{m_{2}}^{+} \alpha_{m_{2}}^{*}\right|\right) .
$$

The corresponding maximum field enhancement is obtained with the frequency $\omega=\operatorname{Re}\left(\omega_{*}\right)$.

The above theory is derived for the diffraction problem with a fixed real wavenumber $\beta$. If there is a $c B I C$ in a band of resonant modes, it is important to understand how field enhancement varies with $\beta$ (or incident angle). Let $u_{\diamond}(\mathbf{r}), \omega_{\diamond}$ and $\beta_{\diamond}$ be the wave field, frequency, and wavenumber of the $\mathrm{cBIC}$, respectively. For the resonant mode with wavenumber $\beta$, we denote its complex frequency by $\omega_{*}(\beta)$, its total $Q$ factor by $Q_{\text {tot }}(\beta)$, etc. As $\beta \rightarrow \beta_{\diamond}$, we have $\omega_{*}(\beta) \rightarrow \omega_{\diamond}, Q_{\mathrm{rad}}(\beta) \rightarrow \infty$, and $Q_{\text {dis }}(\beta) \rightarrow Q_{\text {dis }}\left(\beta_{\diamond}\right)<\infty$. If formula (22) [with $\eta, Q_{\mathrm{rad}}$ and $Q_{\text {dis }}$ all depending on $\left.\beta\right]$ is applicable, the maximum of $\eta(\beta)$ can be found approximately by replacing $Q_{\text {dis }}(\beta)$ by the constant $Q_{\text {dis }}\left(\beta_{\diamond}\right)$. This gives rise to a wavenumber $\beta_{c}$, such that $Q_{\mathrm{rad}}\left(\beta_{c}\right)=Q_{\mathrm{dis}}\left(\beta_{c}\right) \approx Q_{\mathrm{dis}}\left(\beta_{\diamond}\right)$. This is the so-called critical coupling condition. The field enhancement is maximized approximately at this critical wavenumber $\beta_{c}$, and

$$
\eta\left(\beta_{c}\right) \sim\left[Q_{\mathrm{dis}}\left(\beta_{\diamond}\right)\right]^{1 / 2} \sim \frac{L}{\left[\int_{\Omega_{d}} \varepsilon_{i}(\mathbf{r})\left|u_{\diamond}(\mathbf{r})\right|^{2} d \mathbf{r}\right]^{1 / 2}}
$$

It should be emphasized that, strictly speaking, the above is only valid for incident waves with frequency $\omega=\operatorname{Re}\left[\omega_{*}\left(\beta_{c}\right)\right]$ and with coefficients satisfying Eq. (20).

\section{NUMERICAL EXAMPLES}

To validate the analytic results derived in the previous section, we present a few numerical examples for diffraction problems of periodic arrays of cylinders with single and multiple propagating diffraction orders. As shown in Fig. 1, the periodic arrays consist of circular or triangular
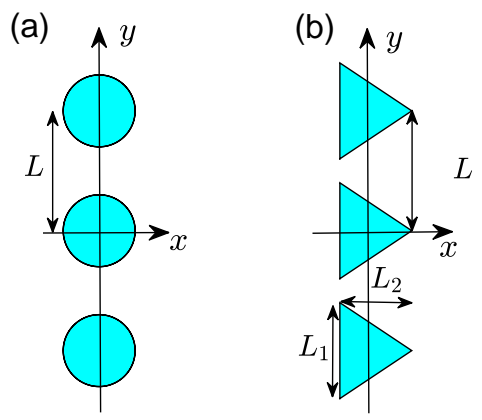

FIG. 1. (a): A periodic array of circular cylinders with radius a. (b): A periodic array of triangular cylinders with base $L_{1}$ and height $L_{2}$.

cylinders (with dielectric constant $\varepsilon_{1}$ ) surrounded by air (with dielectric constant $\varepsilon_{0}=1$ ). The radius of the circular cylinders is $a$. The cross section of a triangular cylinder is an isosceles triangle with base $L_{1}=0.8 L$ and height $L_{2}=0.6 L$, where $L$ is the period in $y$. In Table $\llbracket$ we list 


\begin{tabular}{c|c|c|c|c}
\hline $\mathrm{cBIC}$ & $a / L$ & $\beta_{\diamond}$ & $\omega_{\diamond} L /(2 \pi c)$ & $\mathbb{Z}_{0}$ \\
\hline 1 & 0.3 & 0 & $0.7718567-3.03 \times 10^{-6} i$ & $\{0\}$ \\
\hline 2 & 0.2017 & $\pi / L$ & $1.0935834-4.25 \times 10^{-6} i$ & $\{-1,0\}$ \\
\hline 3 & 0.3194 & 0 & $1.5717916-6.29 \times 10^{-6} i$ & $\{-1,0,1\}$ \\
\hline 4 & N.A. & 0 & $0.8971846-3.40 \times 10^{-6} i$ & $\{0\}$ \\
\hline
\end{tabular}

TABLE I. Complex BICs in lossy periodic arrays of cylinders with $\varepsilon_{1}=12+0.0001 i$. The first three cBICs exist in the array of circular cylinders with radius $a$. The fourth cBIC exists in the the array of triangular cylinders. $\beta_{\diamond}$ and $\omega_{\diamond}$ are the Bloch wavenumber and complex frequency of the cBICs. $\mathbb{Z}_{0}$ is the integer set for open diffraction channels.

four $\mathrm{cBICs}$ for the periodic arrays with $\varepsilon_{1}=12+0.0001$. The first three and the fourth cBICs exist in the periodic arrays of circular and triangular cylinders, respectively. The second and third cBICs have multiple open radiation channels $\left(\mathbb{Z}_{0} \neq\{0\}\right)$, and are found by tuning the radius of the cylinders [22]. The first and fourth $\mathrm{cBIC}$ are fully symmetry-protected. The field patterns of these four cBICs are shown in Fig. 2 .
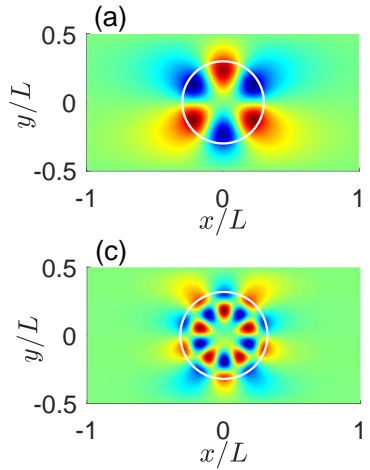

(b)

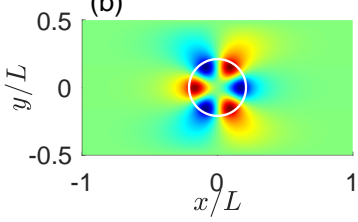

(d)

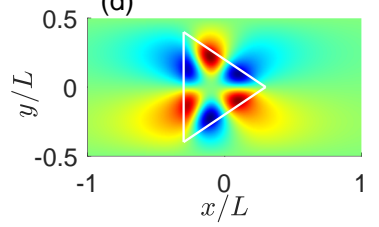

FIG. 2. Field patterns $(\operatorname{Re}(u))$ of the four cBICs listed in Table (a)-(d): cBICs 1 to 4 , respectively.

For cBIC 1, the zeroth diffraction order is the only open radiation channel. Since the structure is symmetric in $x$, the radiation channels in the left and right sides can be considered as the same. In Fig. 3(a), we show $Q_{\text {tot }}, Q_{\text {dis }}$ and $Q_{\text {rad }}$ for the resonant mode near the cBIC as functions of $\beta$. As $\beta \rightarrow \beta_{\diamond}=0, Q_{\mathrm{rad}} \rightarrow \infty$, and $Q_{\text {dis }}$ converges to a constant $\left(\approx 1.27 \times 10^{5}\right)$. The critical coupling condition is satisfied at Bloch wavenumber $\beta_{c} \approx 0.0074(2 \pi / L)$. The corresponding resonant frequency is $\omega_{*} \approx 0.7718519-6.05 \times 10^{-6} i(2 \pi c / L)$. Since cBIC 1 is even in $x$, the nearby resonant mode is also even in $x$, and thus $c_{0}^{+}=c_{0}^{-}$. To maximize field enhancement, we choose an incident wave with frequency $\omega=\operatorname{Re}\left(\omega_{*}\right)$ and coefficients $a_{0}^{+}=a_{0}^{-}=1$. Using the numerical solutions for different $\beta$, we evaluate the field enhancement $\eta$ and its approximation $|C / \delta|$ following Eqs. (18) and (17), respectively. The computed $\eta,|C / \delta|$ and $\sqrt{Q_{\text {rad }}} Q_{\text {dis }} /\left(Q_{\text {rad }}+Q_{\text {dis }}\right)$ are shown in Fig. B (b) as functions of $Q_{\mathrm{rad}}$ (which is related to $\beta$ ). It can be seen that $\eta$ is well approximated by $|C / \delta|$, and is indeed
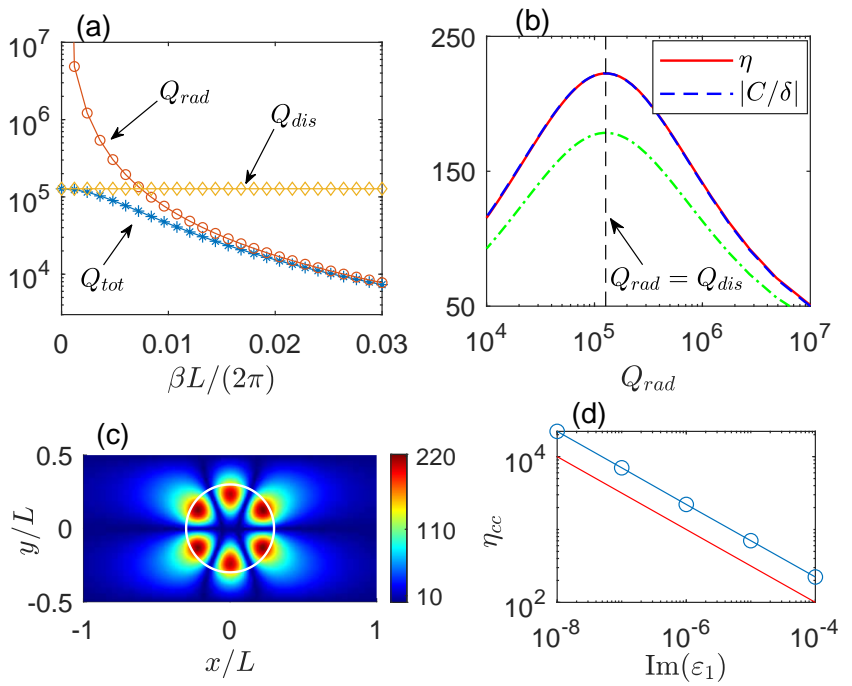

FIG. 3. Field enhancement near cBIC 1. (a): $Q_{\text {tot }}, Q_{\text {rad }}$ and $Q_{\mathrm{dis}}$ of the resonant mode as functions of $\beta$. (b): Field enhancement $\eta$ (red solid curve), $|C / \delta|$ (blue dashed curve), and $\sqrt{Q_{\mathrm{rad}}} Q_{\text {dis }} /\left(Q_{\mathrm{rad}}+Q_{\mathrm{dis}}\right)$ (green dash-dot curve), as functions of $Q_{\mathrm{rad}}$. (c): Magnitude of the diffraction solution at critical coupling, i.e., $|u|$ for $\beta=\beta_{c}$. (d) Field enhancement at critical coupling $\eta\left(\beta_{c}\right)$ ("o") for different values of $\operatorname{Im}\left(\varepsilon_{1}\right)$. The solid red line is the reference line for $1 / \sqrt{\operatorname{Im}\left(\varepsilon_{1}\right)}$.

proportional to $\sqrt{Q_{\mathrm{rad}}} Q_{\mathrm{dis}} /\left(Q_{\mathrm{rad}}+Q_{\mathrm{dis}}\right)$. As indicated by the vertical line in Fig. 3(b), the field enhancement reaches the maximum $\eta\left(\beta_{c}\right) \approx 223$ when the critical coupling condition is satisfied. The corresponding diffraction solution is shown in Fig. 3(c). The above numerical results are obtained for the fixed $\varepsilon_{1}=12+0.0001 i$. Equation (26) indicates that field enhancement is proportional to $1 / \sqrt{\operatorname{Im}\left(\varepsilon_{1}\right)}$, if $\operatorname{Im}\left(\varepsilon_{1}\right)$ is a constant. To validate this result, we keep $\operatorname{Re}\left(\varepsilon_{1}\right)$ fixed and calculate $\eta\left(\beta_{c}\right)$ for different values of $\operatorname{Im}\left(\varepsilon_{1}\right)$. The results are shown in Fig. 3(d).

For cBIC 2, there are two radiation channels (diffraction orders $m=0$ and -1 ) in each side of the periodic array. Due to the reflection symmetry in $x$, the radiation channels in the left and right sides are identical. The symmetry in $y$ is also important to this cBIC. If a resonant mode is even in $y$ and has a Bloch wavenumber $\beta=\pi / L$, then $c_{0}^{ \pm}=c_{-1}^{ \pm}$. For the array of circular cylinders, such a resonant mode with $\beta=\pi / L$ becomes a cBIC (with $c_{0}^{ \pm}=c_{-1}^{ \pm}=0$ ), if the radius is tuned to $a=0.2017 L$. For this $a$, we consider resonant modes with $\beta$ near $\beta_{\diamond}=\pi / L$, and show $Q_{\text {tot }}, Q_{\text {dis }}$

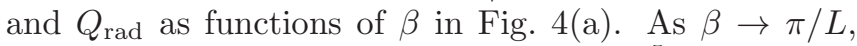
$Q_{\text {dis }}$ converges to a constant $\left(\approx 1.28 \times 10^{5}\right)$. The critical coupling condition is satisfied at the Bloch wavenumber $\beta_{c} \approx 0.4932(2 \pi / L)$. The corresponding resonant frequency is $\omega_{*} \approx 1.0935827-8.53 \times 10^{-6} i(2 \pi c / L)$. For an incident wave with frequency $\omega=\operatorname{Re}\left(\omega_{*}\right)$ and coefficients given by Eq. (20), we solve the diffraction problem, and show field enhancement $\eta$, its approximation $|C / \delta|$ and $\sqrt{Q_{\mathrm{rad}}} Q_{\mathrm{dis}} /\left(Q_{\mathrm{rad}}+Q_{\mathrm{dis}}\right)$, as functions of $Q_{\text {rad }}$, in Fig. $4(\mathrm{~b})$. For $\beta=\beta_{c}$, field enhancement 

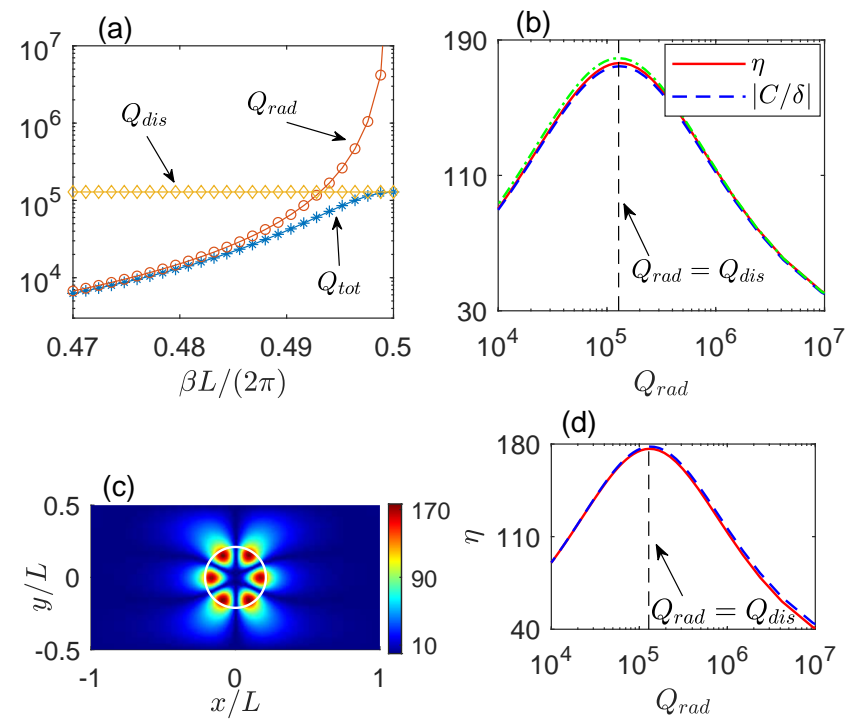

FIG. 4. Field enhancement near cBIC 2. (a): $Q_{\text {tot }}, Q_{\text {rad }}$ and $Q_{\text {dis }}$ of the resonant mode as functions of $\beta$. (b): Field enhancement $\eta$ (red solid curve), $|C / \delta|$ (blue dashed curve), and $\sqrt{Q_{\mathrm{rad}}} Q_{\mathrm{dis}} /\left(Q_{\mathrm{rad}}+Q_{\mathrm{dis}}\right)$ (green dash-dot curve), as functions of $Q_{\mathrm{rad}}$. (c): Magnitude of the diffraction solution at critical coupling, i.e., $|u|$ for $\beta=\beta_{c}$. (d): Field enhancement $\eta(\beta)$ as functions of $Q_{\mathrm{rad}}$, for incident waves with coefficients given by Eq. (20) (red solid curve) and Eq. (27) (blue dashed curve), respectively.

reaches its maximum $\eta\left(\beta_{c}\right) \approx 176$. The corresponding diffraction solution (i.e. $|u|$ ) is shown in Fig. 廿4(c). For $\beta=\beta_{c}$, the radiation coefficients $c_{0}^{+}$and $c_{-1}^{+}$satisfy $\left|c_{-1}^{+} \alpha_{-1}^{*}\right| /\left|c_{0}^{+} \alpha_{0}^{*}\right| \approx 0.97$. Therefore, according to the theory developed in Sec. III, a larger field enhancement can be obtained if the incident wave is concentrated in the zeroth diffraction order. For example, if the coefficients of the incident wave are given by

$$
\left(a_{0}^{+}, a_{-1}^{+}\right)=-\left(a_{0}^{-}, a_{-1}^{-}\right)=(1,0),
$$

then $\eta\left(\beta_{c}\right) \approx 179>176$. In Fig. 4 (d), we compare the field enhancement $\eta$ as a function of $Q_{\mathrm{rad}}$ (related to $\beta$ ), for incident waves with coefficients given by Eqs. (20) and (27), respectively.

The third cBIC exist in the frequency range with three open radiation channels (diffraction orders $m=0$ and \pm 1 ) in each side of the structure. The reflection symmetry in $x$ ensures that the radiation channels in the left and right are identical. The reflection symmetry in $y$ is again important to the existence of cBIC 3. Notice that the electric field of cBIC 3 is odd in $y$. In any periodic array of circular cylinders, a resonant mode with $\beta=0$ and an odd-in- $y$ field must have $c_{0}^{ \pm}=0$ and $c_{1}^{ \pm}=-c_{-1}^{ \pm}$. For the given $\varepsilon_{1}$, as $a$ is tuned to $0.3194 L$, the resonant mode becomes cBIC 3 with $c_{1}^{ \pm}=-c_{-1}^{ \pm}=0$.

Like the previous two examples, we calculate the resonant modes near cBIC 3 for $\beta$ near $\beta_{\diamond}=0$, and show $Q_{\text {tot }}, Q_{\text {rad }}$ and $Q_{\text {dis }}$ in Fig. 5(a). The limit of $Q_{\text {dis }}$ (as $\beta \rightarrow 0)$ is approximately $1.25 \times 10^{5}$. The criti-
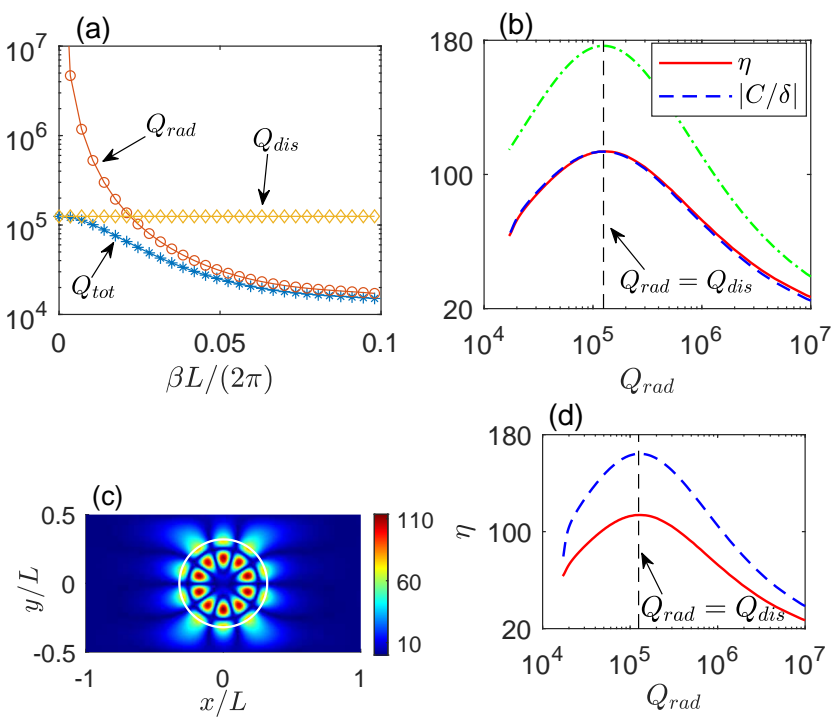

FIG. 5. Field enhancement near cBIC 3. (a): $Q_{\text {tot }}, Q_{\text {rad }}$ and $Q_{\text {dis }}$ of the resonant mode as functions of $\beta$. (b): Field enhancement $\eta$ (red solid curve), $|C / \delta|$ (blue dashed curve), and $\sqrt{Q_{\mathrm{rad}}} Q_{\mathrm{dis}} /\left(Q_{\mathrm{rad}}+Q_{\mathrm{dis}}\right)$ (green dash-dot curve), as functions of $Q_{\mathrm{rad}}$. (c): Magnitude of the diffraction solution at critical coupling, i.e. $|u|$ for $\beta=\beta_{c}$. (d): Field enhancement $\eta(\beta)$ as functions of $Q_{\mathrm{rad}}$, for incident waves with coefficients given by Eq. (20) (red solid curve) and Eq. (28) (blue dashed curve), respectively.

cal coupling condition is satisfied at Bloch wavenumber $\beta_{c} \approx 0.0221(2 \pi / L)$, and the corresponding resonant frequency is $\omega_{*}\left(\beta_{c}\right) \approx 1.5718020-1.26 \times 10^{-5} i(2 \pi c / L)$. For each $\beta$, we solve the diffraction problem for an incident wave with frequency $\omega=\operatorname{Re}\left[\omega_{*}(\beta)\right]$ and coefficients given by Eq. (20), and show field enhancement $\eta$, its approximation $|C / \delta|$, and $\sqrt{Q_{\mathrm{rad}}} Q_{\mathrm{dis}} /\left(Q_{\mathrm{rad}}+Q_{\mathrm{dis}}\right)$, in Fig. [5(b). The maximum field enhancement is $\eta\left(\beta_{c}\right) \approx 114$. The corresponding diffraction solution is shown in Fig. 5 (c). For $\beta=\beta_{c}$, the radiation coefficients of the resonant mode satisfy $\left|\alpha_{0}^{*} c_{0}^{+}\right|:\left|\alpha_{-1}^{*} c_{-1}^{-}\right|:\left|\alpha_{1}^{*} c_{1}^{-}\right| \approx 1: 0.35: 0.34$. Therefore, a larger field enhancement can be obtained, if incident wave consists of a single plane wave in the zeroth diffraction order in each side of the periodic array. For the incident wave with coefficients

$$
\left(a_{0}^{+}, a_{-1}^{+}, a_{1}^{+}\right)=\left(a_{0}^{-}, a_{-1}^{-}, a_{1}^{-}\right)=(1,0,0),
$$

we obtain the field enhancement $\eta\left(\beta_{c}\right) \approx 164>114$. To see the difference for $\beta \neq \beta_{c}$, we compare the field enhancement $\eta(\beta)$ for incident waves given by Eqs. (20) and (28), respectively, in Fig. 5 (d).

The fourth cBIC has only a single radiation channel (diffraction order $m=0$ ) in each side of the structure, but the left and right channels are different due to the lack of symmetry in $x$. In Fig. 6(a), we show $Q_{\text {tot }}, Q_{\text {dis }}$ and $Q_{\text {rad }}$ as functions of $\beta$ for resonant modes near cBIC 4. The limit of $Q_{\mathrm{dis}}$, as $\beta \rightarrow \beta_{\diamond}=0$, is approximately $1.32 \times 10^{5}$. The critical coupling condition is satisfied as $\beta_{c} \approx 0.0237(2 \pi / L)$. The corresponding resonant fre- 

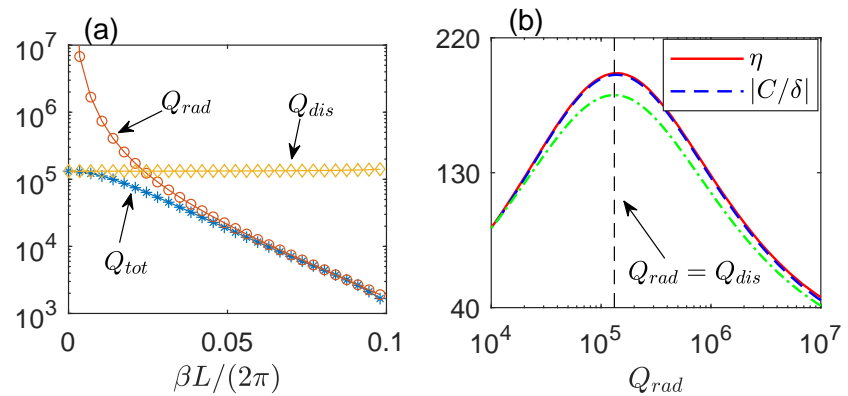

(d)
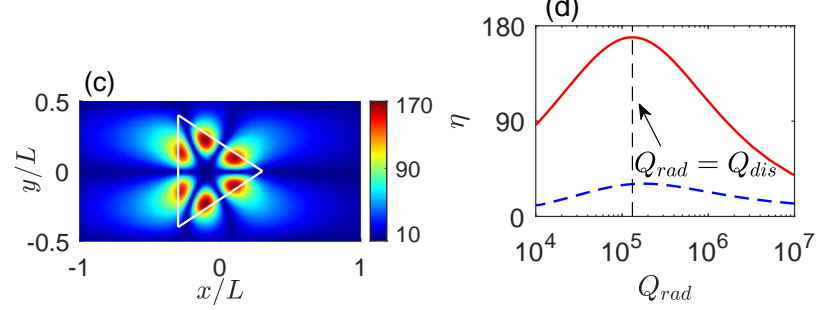

FIG. 6. Field enhancement near cBIC 4. (a) $Q_{\text {tot }}, Q_{\text {rad }}$ and $Q_{\text {dis }}$ of the resonant mode as functions of $\beta$. (b): Field enhancement $\eta$ (red solid curve) and $|C / \delta|$ (blue dashed curve) for the incident wave with coefficients satisfying Eq. (29), and $\sqrt{Q_{\mathrm{rad}}} Q_{\mathrm{dis}} /\left(Q_{\mathrm{rad}}+Q_{\mathrm{dis}}\right)$ (green dash-dot curve), as functions of $Q_{\mathrm{rad}}$. (c): Magnitude of the diffraction solution at critical coupling, i.e., $|u|$ for $\beta=\beta_{c}$. (d): Field enhancement $\eta$ as functions of $Q_{\mathrm{rad}}$, for incident waves with coefficients $\left(a_{0}^{+}, a_{0}^{-}\right)=(1,0)$ (red solid curve) and $(0,1)$ (blue dashed curve), respectively.

quency is $\omega_{*} \approx 0.8970943-6.77 \times 10^{-6} i(2 \pi c / L)$, and the left and right radiation coefficients satisfy $\left|c_{0}^{-} / c_{0}^{+}\right| \approx 0.15$. Since there is only one propagating diffraction order, Eq. (20) implies that $\left(a_{0}^{+}, a_{0}^{-}\right)$is proportional to $\left(\bar{c}_{0}^{+}, \bar{c}_{0}^{-}\right)$, but a larger field enhancement can be achieved if $a_{0}^{ \pm}$satisfy

$$
\left|a_{0}^{ \pm}\right|=1, \quad\left|a_{0}^{-} c_{0}^{-}+a_{0}^{+} c_{0}^{+}\right|=\left|c_{0}^{-}\right|+\left|c_{0}^{+}\right| .
$$

In Fig. 6(b), we show field enhancement $\eta(\beta)$, its approximation $|C / \delta|$, and $\sqrt{Q_{\mathrm{rad}}} Q_{\mathrm{dis}} /\left(Q_{\mathrm{rad}}+Q_{\mathrm{dis}}\right)$, as functions of $Q_{\mathrm{rad}}$, for an incident wave with frequency $\omega=\operatorname{Re}\left[\omega_{*}(\beta)\right]$ and coefficients satisfying Eq. (29). It can be seen that $\eta$ is well approximated by $|C / \delta|$, but is not proportional to $\sqrt{Q_{\mathrm{rad}}} Q_{\mathrm{dis}} /\left(Q_{\mathrm{rad}}+Q_{\mathrm{dis}}\right)$. The maximum field enhancement is approximately $\eta\left(\beta_{c}\right)=195$. The corresponding diffraction solution is shown in Fig. 6(c). In Fig. 6(d), we show the field enhancement $\eta(\beta)$ onesided incident waves with coefficients $\left(a_{0}^{+}, a_{0}^{-}\right)=(1,0)$ (red solid curve) and $(0,1)$ (blue dashed curve), respectively. The field enhancement is clearly dominated by the right incident wave.

\section{CONCLUSION}

In this paper, we studied resonant field enhancement for diffraction problems of $2 \mathrm{D}$ lossy periodic structures based on a rigorous perturbation method. The approximation $\eta \approx|C / \delta|$ is valid for any incident wave with any frequency $\omega$ near the complex resonant frequency $\omega_{*}$, and is highly accurate as shown in the numerical examples. Equation (22) is a simple scaling law between the field enhancement $\eta$ and the radiation and dissipation $Q$ factors, but if the resonant mode has multiple radiation channels, Eq. (22) is only valid for specific incident waves with coefficients given by Eq. (20) and a frequency $\omega$ exactly equals to the real part of $\omega_{*}$, and it does not give the largest field enhancement. In fact, we showed that the largest field enhancement is obtained when the incident wave consists of a single plane wave in each side of the periodic structure with coefficients satisfying Eqs. (23) and (24). We also analyzed the dependence of field enhancement $\eta$ on Bloch wavenumber $\beta$, assuming the periodic structure supports a cBIC. Consistent with existing studies, the field enhancement is approximately maximized at the wavenumber $\beta_{c}$ such that radiation and dissipation $Q$ factors are equal (the critical coupling condition).

Our theoretical results should be useful in applications where a large field enhancement is important, especially when the related resonant mode has multiple radiation channels. Although our theory is developed only for structures with a one-dimensional periodicity, it can be extended to structures with two-dimensional periodicity.

\section{ACKNOWLEDGEMENT}

The authors acknowledge support from the Natural Science Foundation of Chongqing, China (Grant No. cstc2019jcyj-msxmX0717), and the Research Grants Council of Hong Kong Special Administrative Region, China (Grant No. CityU 11305518).

\section{APPENDIX A}

Let $u$ be a resonant mode with a real Bloch wavenumber $\beta$ and a complex resonant frequency $\omega$. Multiplying both sides of Eq. (2) by $\bar{u}$ and integrating the result on domain $\Omega_{d}$, we have

$$
\int_{\partial \Omega_{d}} \bar{u} \frac{\partial u}{\partial \nu} d s-\int_{\Omega_{d}} \nabla \bar{u} \cdot \nabla u d \mathbf{r}+k^{2} \int_{\Omega_{d}} \varepsilon(\mathbf{r})|u|^{2} d \mathbf{r}=0
$$

where $\partial \Omega_{d}$ is the boundary of $\Omega_{d}$ and $\nu$ is the outward unit normal vector of $\Omega_{d}$. In Appendix A of Ref. [17], it is proved that

$$
\int_{\partial \Omega_{d}} \bar{u} \frac{\partial u}{\partial \nu} d s=i L \sum_{m=-\infty}^{\infty} \alpha_{m}\left(\left|c_{m}^{+}\right|^{2}+\left|c_{m}^{-}\right|^{2}\right) .
$$


Taking the imaginary parts of Eqs. (A1) and (A2), we obtain

$$
\begin{aligned}
& L \sum_{m=-\infty}^{\infty} \operatorname{Re}\left(\alpha_{m}\right)\left(\left|c_{m}^{+}\right|^{2}+\left|c_{m}^{-}\right|^{2}\right)+\operatorname{Re}\left(k^{2}\right) \int_{\Omega_{d}} \varepsilon_{i}(\mathbf{r})|u|^{2} d \mathbf{r} \\
& +\operatorname{Im}\left(k^{2}\right) \int_{\Omega_{d}} \varepsilon_{r}(\mathbf{r})|u|^{2} d \mathbf{r}=0 .
\end{aligned}
$$

As shown in Appendix A of Ref. [17], the above leads to

$$
L \sum_{m \notin \mathbb{Z}_{0}} \operatorname{Re}\left(\alpha_{m}\right)\left(\left|c_{m}^{+}\right|^{2}+\left|c_{m}^{-}\right|^{2}\right)=\operatorname{Im}\left(k^{2}\right) \varepsilon_{0} \int_{\Omega_{e}}\left|u_{e}\right|^{2} d \mathbf{r} .
$$

Therefore, Eq. A3 gives rise to

$$
\begin{aligned}
\operatorname{Im}\left(k^{2}\right) & =-\frac{\operatorname{Re}\left(k^{2}\right) \int_{\Omega_{d}} \varepsilon_{i}(\mathbf{r})|u|^{2} d \mathbf{r}}{F} \\
& -\frac{L \sum_{m \in \mathbb{Z}_{0}} \operatorname{Re}\left(\alpha_{m}\right)\left(\left|c_{m}^{+}\right|^{2}+\left|c_{m}^{-}\right|^{2}\right)}{F},
\end{aligned}
$$

where $F$ is defined in Sec. III Notice that

$$
\operatorname{Im}\left(k^{2}\right)=2 \operatorname{Re}(k) \operatorname{Im}(k)
$$

we have

$$
\begin{aligned}
\frac{1}{Q_{t o t}} & =-\frac{\operatorname{Im}\left(k^{2}\right)}{[\operatorname{Re}(k)]^{2}}=\frac{\operatorname{Re}\left(k^{2}\right) \int_{\Omega_{d}} \varepsilon_{i}(\mathbf{r})|u|^{2} d \mathbf{r}}{[\operatorname{Re}(k)]^{2} F} \\
& -\frac{L \sum_{m \in \mathbb{Z}_{0}} \operatorname{Re}\left(\alpha_{m}\right)\left(\left|c_{m}^{+}\right|^{2}+\left|c_{m}^{-}\right|^{2}\right)}{[\operatorname{Re}(k)]^{2} F} .
\end{aligned}
$$

The first and second terms on the right hand side of Eq. (A5) gives to Eqs. (8) and (9), respectively.

\section{APPENDIX B}

Let $u_{*}$ be a resonant mode with a real Bloch wavenumber $\beta$ and a complex frequency $\omega_{*}$, and be expanded as in Eq. (41) with $\alpha_{m}$ replaced by $\alpha_{m}^{*}=\sqrt{k_{*}^{2} \varepsilon_{0}-\beta_{m}^{2}}$ for $k_{*}=\omega_{*} / c$. Since the structure has a reflection symmetry in $y, v_{*}=u_{*}(x,-y)$ is a resonant mode for Bloch wavenumber $-\beta$ and the same complex frequency $\omega_{*}$, and

$$
v_{*}=u_{*}(x,-y)=\sum_{m=-\infty}^{\infty} c_{m}^{ \pm} e^{i\left[-\beta_{m} y \pm \alpha_{m}^{*}(x \mp d)\right]} .
$$

For the incident wave $u^{(i n)}$ given in Eq. (14), the diffraction solution $u$ satisfies Eq. (2) and boundary condition

$$
\pm \frac{\partial u}{\partial x}=\mathcal{B} u-2 i \sum_{m \in \mathbb{Z}_{0}} \alpha_{m} a_{m}^{ \pm} e^{i \beta_{m} y}, \quad x= \pm d
$$

where $\mathcal{B}$ is the operator defined in Eq. (24) of Ref. [17]. Inserting expansion (16) into Eq. (2) and boundary condition (B1), and comparing the coefficient of $\delta^{j}$ for $j \geq$ -1 , we obtain the equation

$$
\partial_{x}^{2} u_{0}+\partial_{y}^{2} u_{0}+k_{*}^{2} \varepsilon u_{0}=-2 C k_{*} \varepsilon u_{*}
$$

and boundary condition

$$
\pm \frac{\partial u_{0}}{\partial x}-\mathcal{B}_{*} u_{0}=C \mathcal{B}_{1} u_{*}-2 i \sum_{m \in \mathbb{Z}_{0}} \alpha_{m}^{*} a_{m}^{ \pm} e^{i \beta_{m} y}, \quad x= \pm d
$$

for $u_{0}$, where $\mathcal{B}_{*}$ and $\mathcal{B}_{1}$ are operators defined in Ref. [17].

Multiplying Eq. (B2) by $v_{*}$ and integrating the result on domain $\Omega_{d}$, we have

$$
\int_{\partial \Omega_{d}}\left(v_{*} \frac{\partial u_{0}}{\partial \nu}-u_{0} \frac{\partial v_{*}}{\partial \nu}\right) d s=-2 C k_{*} \int_{\Omega_{d}} \varepsilon v_{*} u_{*} d \mathbf{r} .
$$

Following the procedure given in Appendix B of Ref. [17], we can show that

$$
\begin{aligned}
& \int_{\partial \Omega_{d}}\left(v_{*} \frac{\partial u_{0}}{\partial \nu}-u_{0} \frac{\partial v_{*}}{\partial \nu}\right) d s+2 i L \sum_{m \in \mathbb{Z}_{0}}\left(a_{m}^{+} c_{m}^{+}+a_{m}^{-} c_{m}^{-}\right) \alpha_{m}^{*} \\
& =i L k_{*} C \sum_{m=-\infty}^{\infty} \frac{\left(c_{m}^{+}\right)^{2}+\left(c_{m}^{-}\right)^{2}}{\alpha_{m}^{*}} .
\end{aligned}
$$

Inserting Eq. (B3) into Eq. (B4) and noticing that $v_{*}=$ $u_{*}(x,-y)$, we obtain Eq. (17).
[1] J. Homola, S. S. Yee, and G. Gauglitz, "Surface plasmon resonance sensors: Review," Sens. Actuators B Chem. 54, 3-15 (1999).

[2] X. Fan, I. M. White, S. I. Shopova, H. Zhu, J. D. Suter, and Y. Sun, "Sensitive optical biosensors for unlabeled targets: A review," Anal. Chim. Acta 620, 8-26 (2008).

[3] Y. Zhang, Y. Zhao, and R. Lv, "A review for optical sensors based on photonic crystal cavities," Sens. Actuators A Phys. 233, 374-389 (2015).

[4] S. Romano, A. Lamberti, M. Masullo, E. Penzo, S. Cabrini, I. Rendina, and V. Mocella, "Optical biosensors based on photonic crystals supporting bound states in the continuum," Materials 11, 526 (2018).
[5] F. Yesilkoy, E. R. Arvelo, Y. Jahani, M. Liu, A. Tittl, V. Cevher, Y. Kivshar, and H. Altug, "Ultrasensitive hyperspectral imaging and biodetection enabled by dielectric metasurfaces," Nat. Photon. 13, 390-396 (2019).

[6] S. Romano, M. Mangini, E. Penzzo, S. Cabrini, A. Chiara De Luca, I. Rebdina, V. Mocella, and G. Zito, "Ultrasensitive surface refractive index imaging based on quasi-bound states in the continuum," ACS Nano 14(11), 15417-15427 (2020).

[7] S. A. Maier, Plasmonics: Fundamentals and Applications (Springer 2007).

[8] S. Kim, J. Jin, Y.-J. Kim, I.-Y. Park, Y. Kim, and S.-W. Kim, "High-harmonic generation by resonant plasmon 
field enhancement," Nature 453, 757-760 (2008)

[9] L. Yuan and Y. Y. Lu, "Diffraction of plane waves by a periodic array of nonlinear circular cylinders," Phys. Rev. A 94, 013852 (2016).

[10] L. Yuan and Y. Y. Lu, "Strong resonances on periodic arrays of cylinders and optical bistability with weak incident waves," Phys. Rev. A 95, 023834 (2017).

[11] K. Koshelev, Y. Tang, K. Li, D.-Y. Choi, G. Li, and Y. Kivshar, "Nonlinear metasurfaces governed by bound states in the continuum," ACS Photonics 6, 1639-1644 (2019).

[12] K. Koshelev, S. Kruk, E. Melik-Gaykazyan, J.-H. Choi, A. Bogdanov, H.-G. Park, and Y. Kivshar, "Subwavelength dielectric resonators for nonlinear nanophotonics," Science 367, 288-292 (2020).

[13] L. Yuan and Y. Y. Lu, "Excitation of bound states in the continuum via second harmonic generations," SIAM J. Appl. Math. 80, 864-880 (2020).

[14] J. W. Yoon, S. H. Song, and R. Magnusson, "Critical field enhancement of asymptotic optical bound states in the continuum," Sci. Rep. 5, 18301 (2015).

[15] V. Mocella and S. Romano, "Giant field enhancement in photonic lattices," Phys. Rev. B 92, 155117 (2015).

[16] E. N. Bulgakov and D. N. Maksimov, "Light enhancement by quasi-bound states in the continuum in dielectric arrays," Opt. Express 25, 14134-14147 (2017).

[17] Z. Hu, L. Yuan, and Y. Y. Lu, "Resonant field enhancement near bound states in the continuum on periodic structures," Phys. Rev. A 101, 043825 (2020).

[18] L. Hsu, F. I. Baida, and D. Ndao, "Local field enhancement using a photonic-plasmonic nanostructure," Opt. Express, 29 (2), 1102-1108 (2021).

[19] M. S. Bin-Alam, O. Reshef, Y. Mamchur, M. Z. Alam, G. Carlow, J. Upham, B. T. Sullivan, J.-M. Menard, M. J. Huttunen, R. W. Boyd, and K. Dolgaleva, "Ultra-high-Q resonances in plasmon metasurfaces," Nature Communications 12, 974 (2021).

[20] C. W. Hsu, B. Zhen, J. Lee, S.-L. Chua, S. G. Johnson, J. D. Joannopoulos, and M. Soljačić, "Observation of trapped light within the radiation continuum," Nature 499, 188-191 (2013).
[21] Y. Yang, C. Peng, Y. Liang, Z. Li, and S. Noda, "Analytical perspective for bound states in the continuum in photonic crystal slabs," Phys. Rev. Lett. 113, 037401 (2014).

[22] E. N. Bulgakov and A. F. Sadreev, "Bloch bound states in the radiation continuum in a periodic array of dielectric rods," Phys. Rev. A 90, 053801 (2014).

[23] Z. Hu and Y. Y. Lu, "Standing waves on two-dimensional periodic dielectric waveguides," J. Opt. 17, 065601 (2015).

[24] L. Yuan and Y. Y. Lu, "Propagating Bloch modes above the lightline on a periodic array of cylinders," J. Phys. B: Atomic, Mol. and Opt. Phys. 50, 05LT01 (2017).

[25] J. Jin, X. Yin, L. Ni, M. Soljačić, B. Zhen, and C. Peng, "Topologically enabled unltrahigh-Q guided resonances robust to out-of-plane scattering," Nature 574, 501-504 (2019).

[26] W. Liu, B. Wang, Y. Zhang, J. Wang, M. Zhao, F. Guan, X. Liu, L. Shi, and J. Zi, "Circularly Polarized States Spawning from Bound States in the Continuum," Phys. Rev. Lett. 123, 116104 (2019).

[27] Q. Song, J. Hu, S. Dai, C. Zheng, D. Han, J. Zi, Z. Q. Zhang, and C. T. Chan, "Coexistence of a new type of bound state in the continuum and a lasing threshold mode induced by PT symmetry," Science Advances 6, eabc1160 (2020).

[28] T. Yoda and M. Notomi, "Generation and annihilation of topologically protected bound states in the continuum and circularly polarized states by symmetry breaking," Phys. Rev. Lett. 125, 053902 (2020).

[29] Z. Hu, L. Yuan, and Y. Y. Lu, "Bound states with complex frequencies near the continuum on lossy periodic structures," Phys. Rev. A 101, 013806 (2020).

[30] K. Y. Bliokh, Y. P. Bliokh, V. Freilikher, S. Savel'ev, and F. Nori, "Colloquium: Unusual resonantors: plasmonics, metamaterials, and random media," Rev. Mod. Phys. 80, 1201 (2008).

[31] A. Yariv, "Critical coupling and its control in optical waveguide-ring resonantor systems," IEEE Photonics Technol. Lett. 14, 483-485 (2002). 